\title{
Analysis of a Model for the Morphological Structure of Renal Arterial Tree: Fractal Structure
}

\author{
Aurora Espinoza-Valdez, ${ }^{1}$ Francisco C. Ordaz-Salazar, ${ }^{2}$ \\ Edgardo Ugalde, ${ }^{3}$ and Ricardo Femat ${ }^{4}$ \\ ${ }^{1}$ Departamento de Ciencias Computacionales, CUCEI, Universidad de Guadalajara, Avenida Revolución 1500, \\ CP 44430, Guadalajara, JAL, Mexico \\ ${ }^{2}$ Universidad Politécnica de San Luis Potosí, Urbano Villalón 500, CP 78363, La Ladrillera, SLP, Mexico \\ ${ }^{3}$ Instituto de Física UASLP, Universidad Autónoma de San Luis Potosí, Avenida Manuel Nava No. 6., Zona Universitaria, \\ CP 78200, SLP, Mexico \\ ${ }^{4}$ Laboratorio para Biodinámica y Sistemas Alineales, División de Matemáticas Aplicadas, IPICYT, Apartado Postal 3-90, \\ CP 78231, Tangamanga, SLP, Mexico
}

Correspondence should be addressed to Aurora Espinoza-Valdez; aurora.espinoza@cucei.udg.mx

Received 7 March 2013; Accepted 12 June 2013

Academic Editor: Kiwoon Kwon

Copyright ( 2013 Aurora Espinoza-Valdez et al. This is an open access article distributed under the Creative Commons Attribution License, which permits unrestricted use, distribution, and reproduction in any medium, provided the original work is properly cited.

One of the fields of applied mathematics is related to model analysis. Biomedical systems are suitable candidates for this field because of their importance in life sciences including therapeutics. Here we deal with the analysis of a model recently proposed by Espinoza-Valdez et al. (2010) for the kidney vasculature developed via angiogenesis. The graph theory allows one to model quantitatively a vascular arterial tree of the kidney in sense that (1) the vertex represents a vessels bifurcation, whereas (2) each edge stands for a vessel including physiological parameters. The analytical model is based on the two processes of sprouting and splitting angiogeneses, the concentration of the vascular endothelial growth factor (VEGF), and the experimental data measurements of the rat kidneys. The fractal dimension depends on the probability of sprouting angiogenesis in the development of the arterial vascular tree of the kidney, that is, of the distribution of blood vessels in the morphology generated by the analytical model. The fractal dimension might determine whether a suitable renal vascular structure is capable of performing physiological functions under appropriate conditions. The analysis can describe the complex structures of the development vasculature in kidney.

\section{Introduction}

The arterial structure of organs has been the subject of many studies [1-5]. However, not all systems have similar functions; for the organs the purpose of arterial structure is to provide the blood required in the metabolic process and other specific functions. The arterial structure is highly nonuniform because it is determined by reasons of anatomy and local flow requirements [4]. The kidney is one of the most complicated organs in terms of structure and physiology because it is highly vascularized and constitutes the main organ for maintaining chemical balance in blood [6]. The kidney consists of three trees: arterial, venous, and ureter. The arterial vascular tree of the kidney is structured by the renal artery branches into interlobar arteries, arcuate arteries, and interlobular arteries, which are formed by bifurcation [6]. The development of the arterial vascular tree of the kidney can be formed mainly by angiogenesis [1]. The process of angiogenesis is the formation of new blood vessels from preexisting vessels and consists of two different processes: sprouting and splitting. Sprouting refers to the case in which the new branch literally sprouts to some existing branch. In splitting a branch is divided into two new branches [7]. The factors involved at the vascular development are VEGF, reninexpression, ephrins $A$ and $B$, platelet-derived growth factor-B (PDGF-B), Ets family (as Ets-1 and TEL), and angiopoietins 
1-2. The VEGF is an essential regulatory factor for both processes of angiogenesis in the development of renal arterial vascularization [1]. Then, we only consider VEGF, because the other factors increase the complexity of the analytical model.

Zamir and Phipps in [8] studied the morphological characteristics of the rat kidney and found that the branching rules of these vessels are determined by considerations of the angiological function. Subsequently, Zamir generated a model based on L-systems (Lindenmayer System) and incorporated some physiological laws, that is, random parameters [4]. Zamir's results suggest that the arterial structure of the tree is determined mainly by the growth rules of arterial tree branching [4]. The structural morphologic reconstruction of renal vasculature from microcomputed tomography (microCT) images was presented by Nordsletten et. al. The arterial and venous trees of the rat kidney were generated numerically using micro-TC [3]. These morphological data provide a statistical basis from which renal vascular topology can be generated [5]. Graph theory generates branching tree structures incorporating the physiological laws of the renal artery branching through the process of angiogenesis [5]. Many pathological conditions, such as cancers, arteriovenous malformations, and diabetes, induce changes to vessel's morphology or spatial organization. The fractal analysis has been applied to a large variety of healthy or pathological vascular networks [9-14]. Sabo et al. concluded in [15] that the microvessel fractal dimension as a marker of tumor microvascular complexity might provide important pronostic information as well as shed light on the complex interactions between tumor angiogenesis and growth. Moreover, Cross et al. analyzed the fractal dimension of renal angiograms: a normal kidney, a congenitally dysplastic kidney, a kidney with renal artery stenosis, and a kidney with recurrent thromboembolism lesions (see Figure 3 in [16]). The analysis of fractal dimension may allow for characterization of the vascular morphology.

In this paper we extend the results of [5] by analysing mathematically the structure of the arterial vascular tree of the kidney and by incorporating physiological parameters generated from a computational model. In the implementation of the proposed model the branches are represented by the edges of the graph and the ramification point is represented by the nodes [5]. We expect that this representation implemented in computer methodology will help to understand the mechanism involved in biological systems, specifically the development of the renal arterial bifurcation. The analysis provides the mean of the length in each level, and the average width of the arterial vascular tree of the rat kidney is in the range of what is observed in previous experimental and computational models. The fractal dimension depends on the sprouting angiogenesis that appears in the development of the arterial vascular tree of the kidney. The complex structures of the renal arterial tree are consistent with previous fractal analysis of vascular structures [9, 11-14]. These study opens the possibility of a new taxonomy for normal kidneys and for the pathological injuries related to the vascular morphology of the kidneys.

\section{Theoretical Basis}

The representation of the renal arterial vascularization in the computational model is as follows: (1) the arterial vascular tree of the kidney is defined by labeled and oriented graphs; we can identify two objects: the branches (edges) and the branching point (nodes). That is, the edge represents a blood vessel and the vertex is where the angiological stimulus appears. (2) The length and diameter of the new branches are smaller than their parent branches $[5,6]$.

The arterial vascular tree of the kidney down to the interlobular arteries can be structured as follows: renal artery, level 0: interlobar arteries, levels 1-2: arcuate arteries, levels 3-4: interlobular arteries, and levels 5-9. We based in the experiments of Tomanek [3] for the morphological structure of the renal arterial tree. In the model, we develop the arterial vascular tree of the rat kidney. Therefore, the model based on the graph theory for the arterial tree is a binary tree of ten levels and has a total of vessels equal to 1023 . The physiological parameters, and rules are based on parent vessel branch level, the parameters, and the morphological structure of their child branches, and this process deploys the structure of the model. The parameters included in the edges of the binary tree model are $s, C_{g f}, l, d$, and $\theta$ as follows (for details see [5]).

(i) The Type of Angiogenesis: Sprouting or Splitting (s). Angiogenesis is identified by the variable $s$ which can take the values in the set $\left\{a_{b}, a_{p}\right\}$, where $a_{b}$ denotes sprouting angiogenesis and $a_{p}$ denotes splitting angiogenesis. Both sprouting and splitting angiogeneeis generate branching tree structures, which can be represented using graph theory. It is conjectured that the sprouting and splitting processes occur with different probability in the renal arterial tree for the morphology of the kidney. In the proposed computational model this assumption is controlled probabilistically. Sprouting angiogenesis is more probably than splitting angiogenesis because it requires only reorganization of existing endothelial cells (not migration), that is, having less energy requirements [1].

(ii) Concentration of the VEGF in the Vessel $\left(C_{g f}\right)$. Both processes, sprouting and splitting angiogeneses, depend on the regulation of $C_{g f}$ in the pre-existing vessel [1]. While in sprouting angiogenesis it is known that endothelial cells promotes the differentiation, migration, proliferation, and assembly, the effect on splitting angiogenesis is still in research. In the model $C_{g f}$ is generated randomly in a uniform way within the range $[0,35] \mathrm{ng} / \mathrm{mL}$ reported in $[5,17]$.

(iii) Length of the Vessel (l). Here, we focus on determining the value of the lengths in the arterial bifurcations depending of the concentration of the VEGF. Although the effect of $C_{g f}$ over length for the splitting angiogenesis is unknown, in the sprouting angiogenesis we consider that the length of the new vessels depends of the $C_{g f}$. We obtain the value of the dimensionless length $l_{e}:\left[C_{g f}, \overline{C_{g f}}\right] \rightarrow\left[l_{\underline{e}}, \overline{l_{e}}\right]$ from 
experimental data; the function which can be approximated for $\mathrm{VEGF}_{121}$ is [5] $l_{e}=0.00878 C_{g f}^{3}$ $0.51326 C_{g f}^{2}+8.52128 C_{g f}+81.12064$. The $l_{e}$ is applied in this model; the length of the vessel $l$ depends on $l_{e}$ and is compared with the length ranges reported in the literature according to the level $[3,5]$. The $l$ formed by splitting is defined by a contraction factor. In the case that $l$ is out of these ranges, the process is repeated finite number of times. If $l$ is still out of range, then it is adjusted to the length of its parent branch.

(iv) Diameter of the Vessel (d). In this work, we do not discuss the value of the diameters in the blood vessels. This is due to the fact that we do not have sufficient information of $C_{g f}$ in $d$ for sprouting and splitting angiogeneses. Then, in sprouting angiogenesis, the diameter of the sprout is adjusted to a factor of the diameter of its parent branch. In splitting angiogenesis the diameter of every new vessel is adjusted to onehalf of the diameter of its parent branch.

(v) Angle of Bifurcation $(\theta)$. In sprouting angiogenesis the angle of bifurcation is adjusted within the range $\pm\left[60^{\circ}, 80^{\circ}\right]$ with respect to its parent branch $[1,18]$. In splitting angiogenesis, one branch is adjusted to $+32.5^{\circ}$ and the other branch to $-32.5^{\circ}[18]$.

The physiological parameters are included in the label of the edge and have the form $\left(s, C_{g f}, l, d, \theta\right)$. The arterial vascular tree of the kidney has vertices with oriented edges in such a way that from each vertex one edge arrives and two edges leave (the orientation symbolizes the circulation of blood flow in arteries of the kidney). The renal arterial vascular tree of the kidney has 10 levels, because we based in the experiments of Tomanek. Therefore, the arterial vascular tree of the kidney has $2^{10}$ nodes. The set of integers to name every node is $\left[0, \ldots, 2^{10}-1\right]$.

An algorithm generates step by step the physiological characteristics of every branch and saves it as labeled edges with the format $\left\{s, C_{g f}, l, d, \theta\right\}$. Just as the tree is generated, the other structure is constructed in which we save the position of every node in $\mathbb{R}^{2}$. The algorithm calculates the position of every node in function of the physiological characteristics of the branches and the position of nodes of its parent branch. The physiological parameters of the root (the initial condition) are determined: $s=a_{b}$ with probability $P_{a b}=$ $\{0.1,0.2,0.3,0.4,0.5\}$ and $s=a_{p}$, where $P_{a p}=1-P_{a b}$, $C_{g f} \in[0,35] \mathrm{ng} / \mathrm{mL}, l=5, d=1, \theta=0$, and they continue in numerical order with the other branches using the information of parent branches. Some characteristics such as $s$ and $C_{g f}$ are generated probabilistically in every step, and the others depend on these parameters, their parent branch, and the rules that were enumerated in the previous section.

\section{Main Results}

3.1. Length and Width. The length of each level into the kidney depends on $a_{b}$ and $a_{p}$; that is, the length for each level $j$ denoted by $l_{j}$ in the arterial vascular tree of the kidney is analytically determined as follows:

$$
l_{j}=l_{00}\left(P_{a b} \lambda_{b}+P_{a p} \lambda_{p}\right)^{j}
$$

where the parameters are defined as in the previous section. Then, the mean length for each segment $j$ is

$$
\begin{array}{cc}
l_{0}=5 \mathrm{~mm}, & l_{1}=3.095 \mathrm{~mm}, \\
l_{2}=1.919 \mathrm{~mm}, & l_{3}=1.191 \mathrm{~mm}, \\
l_{4}=0.741 \mathrm{~mm}, & l_{5}=0.462 \mathrm{~mm}, \\
l_{6}=0.287 \mathrm{~mm}, & l_{7}=0.179 \mathrm{~mm}, \\
l_{8}=0.112 \mathrm{~mm}, & l_{9}=0.070 \mathrm{~mm} .
\end{array}
$$

These data are within the range of rat trees reported in [3]; we compare the results with Nordsletten from depth $j=0$ to $j=9$.

Moreover, the analytical average width denoted by $A_{G_{R}}$ is the root length (renal artery, $j=0$ ) to the leaves of the tree (interlobular arteries, $j=9$ ), and then

$$
A_{G_{R}}=l_{00} \sum_{j=0}^{9}\left(P_{a b} \lambda_{b}+P_{a p} \lambda_{p}\right)^{j},
$$

where $0 \leq j \leq 9 ; l_{00}=5 \mathrm{~mm}$ is the length of the renal artery [5]; $P_{a b}$ is defined in the set $\{0.1,0.2,0.3,0.4,0.5\}$ and $P_{a p}=$ $1-P_{a b} ; \lambda_{b}=0.5$ is the average of the contraction factor for $a_{b}$; and $\lambda_{p}=0.67$ is the contraction factor for $a_{p}$. Then,

$$
\begin{array}{lll}
P_{a b}=0.1, & P_{a p}=0.9, & A_{G_{R}}=14.20 \mathrm{~mm}, \\
P_{a b}=0.2, & P_{a p}=0.8, & A_{G_{R}}=13.59 \mathrm{~mm}, \\
P_{a b}=0.3, & P_{a p}=0.7, & A_{G_{R}}=13.01 \mathrm{~mm}, \\
P_{a b}=0.4, & P_{a p}=0.6, & A_{G_{R}}=12.48 \mathrm{~mm}, \\
P_{a b}=0.5, & P_{a p}=0.5, & A_{G_{R}}=11.99 \mathrm{~mm} .
\end{array}
$$

These data are within the range of rat trees reported in [3]; while we consider 10 levels $[1,5]$, Nordsletten considers 11 levels.

However, if $\lambda_{b} \in[0.3,0.7]$, the average with respect to $\lambda_{b}$ is as follows:

$$
\begin{aligned}
E_{b} & \left(\left(P_{a b} \lambda_{b}+P_{a p} \lambda_{p}\right)^{j}\right) \\
& =\frac{5}{2 P_{a b}} \int_{.3}^{.7}\left(P_{a b} \lambda_{b}+P_{a p} \lambda_{p}\right)^{j} P_{a b} d \lambda_{b} \\
& =\frac{5}{2 P_{a b}(j+1)}\left[\left(.7 P_{a b}+P_{a p} \lambda_{p}\right)^{j+1}-\left(.3 P_{a b}+P_{a p} \lambda_{p}\right)^{j+1}\right] .
\end{aligned}
$$


Let $a=\left(.7 P_{a b}+P_{a p} \lambda_{p}\right)^{j+1}$ and $b=\left(.3 P_{a b}+P_{a p} \lambda_{p}\right)^{j+1}$, and then

$$
\begin{aligned}
A_{G_{R}} & =\frac{5 l_{00}}{2 P_{a b}} \sum_{j=0}^{9}\left(\frac{a^{j+1}}{j+1}-\frac{b^{j+1}}{j+1}\right) \\
\simeq & \frac{5 l_{00}}{2 P_{a b}} \sum_{j=0}^{\infty}\left(\frac{a^{j+1}}{j+1}-\frac{b^{j+1}}{j+1}\right),
\end{aligned}
$$

where

$$
\begin{aligned}
\sum_{j=0}^{\infty} \frac{a^{j+1}}{j+1} & =\sum_{j=0}^{\infty}\left(\int_{0}^{a} x^{j} d x\right) \\
& =\int_{0}^{a}\left(\sum_{j=0}^{\infty} x^{j}\right) d x \quad \text { (by uniform convergence) } \\
& =\int_{0}^{a} \frac{d x}{1-x}=-\left.\ln (1-x)\right|_{0} ^{a}=\ln \left(\frac{1}{1-a}\right) .
\end{aligned}
$$

Similarly, $\sum_{j=0}^{\infty}\left(b^{j+1} / j+1\right)=\ln (1 / 1-b)$, and then

$$
\begin{aligned}
A_{G_{R}} & =\frac{5 l_{00}}{2 P_{a b}} \ln \left(\frac{1-b}{1-a}\right) \\
& =\frac{5 l_{00}}{2 P_{a b}} \ln \left(\frac{1-\left(.3 P_{a b}+P_{a p} \lambda_{p}\right)}{1-\left(.7 P_{a b}+P_{a p} \lambda_{p}\right)}\right) \pm .7^{11},
\end{aligned}
$$

where $\pm .7^{11} \mathrm{~mm}$ is the error.

Substituting the values $l_{00}, P_{a b}, P_{a p}$, and $\lambda_{p}=0.67$,

$$
\begin{array}{lll}
P_{a b}=0.1, & P_{a p}=0.9, & A_{G_{R}}=14.42 \pm .7^{11} \mathrm{~mm}, \\
P_{a b}=0.2, & P_{a p}=0.8, & A_{G_{R}}=13.79 \pm .7^{11} \mathrm{~mm}, \\
P_{a b}=0.3, & P_{a p}=0.7, & A_{G_{R}}=13.23 \pm .7^{11} \mathrm{~mm}, \\
P_{a b}=0.4, & P_{a p}=0.6, & A_{G_{R}}=12.73 \pm .7^{11} \mathrm{~mm}, \\
P_{a b}=0.5, & P_{a p}=0.5, & A_{G_{R}}=12.28 \pm .7^{11} \mathrm{~mm} .
\end{array}
$$

We generate 5000 trees of renal arterial vasculature for different probabilities in $a_{b}$ and $a_{p}$. In Figure 1 the mean \pm SD (standard deviation) width of the kidney has an intersection with these data and previous result where the mean width is $17.454 \pm 6.165 \mathrm{~mm}[3]$.

Therefore, the $A_{G_{R}}$ depends on the process of angiogenesis; that is, $A_{G_{R}}$ is inversely proportional to the probability of the $a_{b}$.

3.2. Fractal Dimension of Kidney Vascular Tree. The fractal dimension $(D)$ quantifies through dimension the ability of an object to occupy a space. Different methods exist and are based on the different definitions of fractal dimension $[9,11-$ $14,19]$.

The analytical fractal dimension can be derived using the following result [19]. Let $\left\{\mathbb{R}^{m} ; w_{1}, w_{2}, \ldots, w_{N}\right\}$ be a hyperbolic

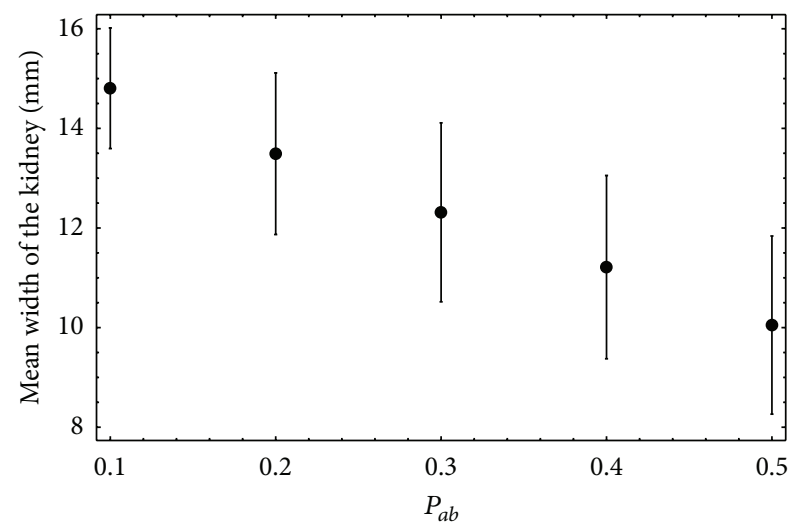

FIGURE 1: The mean \pm SD of width of 5000 trees generated for 5 different probabilities of $a_{b}$ and $a_{p}$.

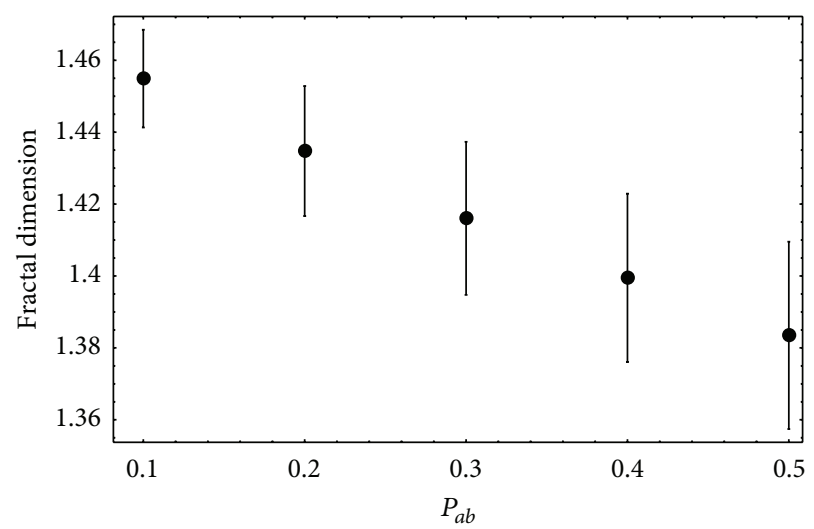

FIGURE 2: The mean \pm SD of fractal dimension for different probabilities of $a_{b}$, where $a_{p}$ has the probability $P_{a p}=1-P_{a b}$.

iterative function system (IFS), and let $A$ denote its attractor. Suppose that $w_{n}$ is a similitude of scaling factor $s_{n}$ for each $n \in\{1,2,3, \ldots, N\}$. If the IFS is totally disconnected or just touching, the attractor has fractal dimension $D(A)$, which is given by the unique solution of

$$
\sum_{n=1}^{N}\left|s_{n}\right|^{D(A)}=1, \quad D(A) \in[0, m] .
$$

If the IFS is overlapping, then $\bar{D} \geq D(A)$, where $\bar{D}$ is the solution of

$$
\sum_{n=1}^{N}\left|s_{n}\right|^{\bar{D}}=1, \quad \bar{D} \in[0, \infty) .
$$

The morphology of arterial vascular tree of the kidney, produced by an IFS, is composed of $n$ applications of contraction with a factor $s_{n}$. Thus, from the subsection of length and width, the contraction factor for the splitting angiogenesis is $\lambda_{p}=0.67$ while for sprouting angiogenesis the average 

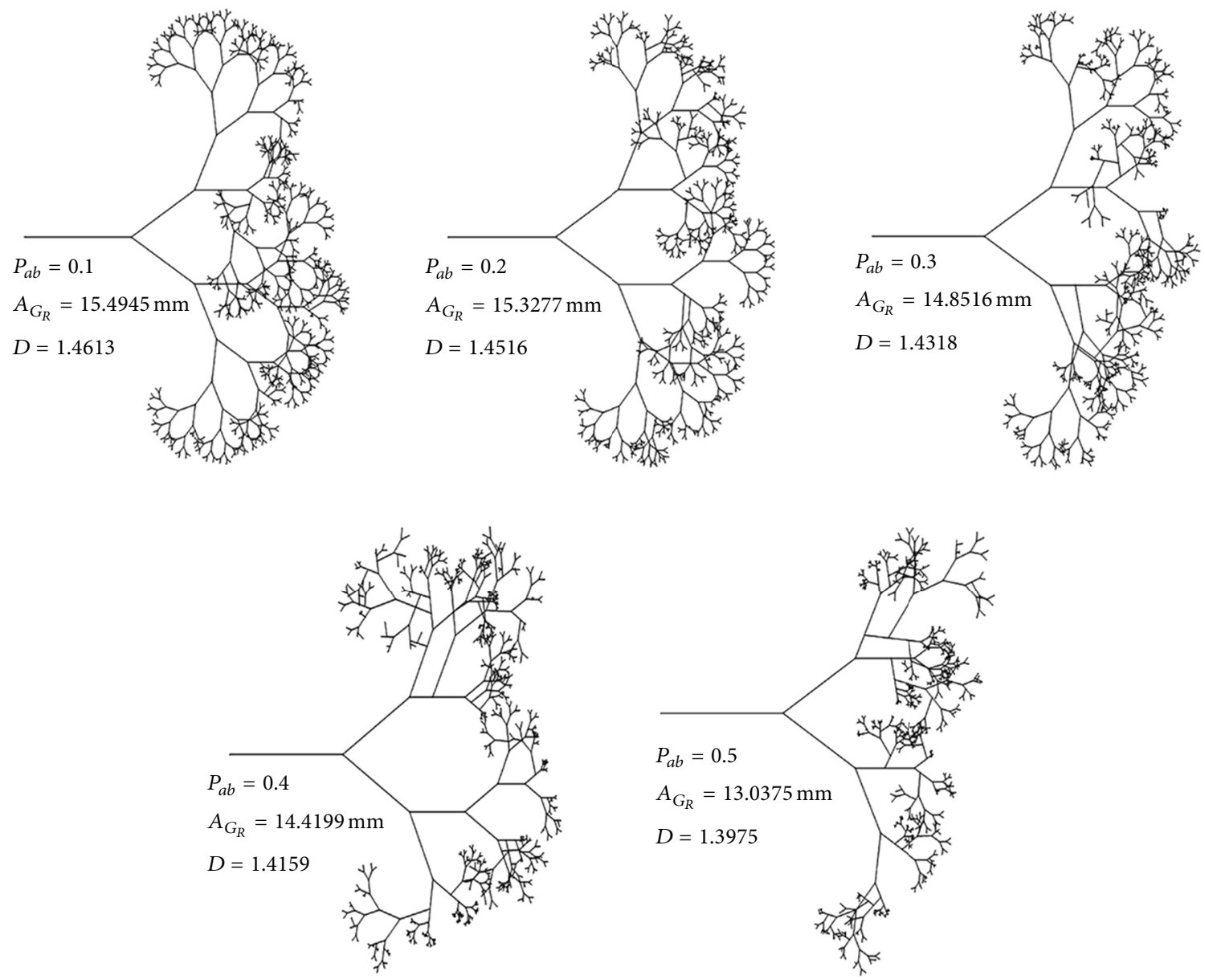

Figure 3: Trees generated with different probabilities in $a_{b}$ and $a_{p}$ (where $P_{a p}=1-P_{a b}$ ), mean width $A_{G_{R}}$ and fractal dimension $D$ of the kidney.

contraction factor is $\lambda_{b}=0.5$. Since we have $P_{a b} \lambda_{b}^{D}+P_{a p} \lambda_{p}^{D}=$ 1 , the analytical fractal dimension of the renal arterial tree is

$$
D=\frac{\ln \left(P_{a p}\right)-\ln \left((1 / 2)-P_{a b} \lambda_{b}^{D}\right)}{-\ln \left(\lambda_{p}\right)},
$$

where $\lambda_{b}$ is generated randomly within the range defined by $\lambda_{b} \in[0.3,0.7]$. In other words, the fractal dimension depends on the number of vessels, the spatial relationships between the vascular components, and the surrounding environment.

3.2.1. Box Counting D of the Arterial Vascular Tree of the Kidney. Additionally, the box counting theorem by Barnsley [19] states that, denoting with $N_{n}\left(G_{R}\right)$ as the number of boxes of side $1 / 2^{n}$,

$$
D=\lim _{n \rightarrow \infty} \frac{\ln \left(N_{n}\left(G_{R}\right)\right)}{\ln \left(2^{n}\right)},
$$

where the arterial vascular tree of the kidney has fractal dimension $D$. The box counting results allow one to compute the rate of change in complexity with scale as well as a measure of hetreogeneity.
Here, we used the box counting theorem for determining the fractal dimension. Figure 2 shows the average of the fractal dimension in each probability for the arterial vascular tree of the kidney. The fractal dimension decreases with the increase in the probability of sprouting in the development of the arterial vascular tree of the kidney, whereas the mean width of the kidney decreases. Then, the fractal dimension of the arterial vascular tree of the kidney depends on the probability of $a_{b}$ and $a_{p}$, that is, the distribution of blood vessels in the morphology generated by graph theory model.

The results suggest that the fractal dimension is inversely proportional to $P_{a b}$. This behavior is congruent in the sense that, by symmetry structure, the arterial vascular tree is bigger for small values of $P_{a b}$; that is, the renal space is covered more efficiently. The complex structures of the vasculature in kidney are consistent with previous studies of vascular structures about their fractal nature [9-16].

Examples derived from graph model using different probabilities in $a_{b}$ and $a_{p}$, mean width and fractal dimension, are shown in Figure 3. The analysis of these responses may allow for characterization of the vascular morphology on the arterial vascular tree of the kidney. 


\section{Conclusions}

The arterial vascular tree of kidney development was analyzed through angiogenesis. The renal arterial vascular tree of the kidney goes down into the interlobular arteries. We generate 5000 trees of renal arterial vasculature for different probabilities on $a_{b}$ and $a_{p}$. The analytical mean length in each level and the average width of the arterial vascular tree of the kidney have an intersection with previous studies. Then, the graph theory allows the vascular tree to model the vascular growth; that is, it generates the ramification of structures arborescent incorporating physiological laws of arterial branching.

In conclusion, the analytical expression of the fractal dimension depends on the number of vessels, the spatial relationships between the vascular components, and the surrounding environment. The arterial vascular arterial tree of the kidney has a fractal dimension which is inversely proportional to the probability of the occurring sprouting angiogenesis. The fractal dimensions determined for the development of the arterial vascular tree of the kidney by box counting may allow for characterization of the vascular morphology. As a conjecture, the fractal dimension might determine whether a suitable renal vascular structure is capable of performing physiological functions under appropriate conditions (hemodynamics). These studies could be expanded to include those pathologies originating from arterial alterations.

\section{References}

[1] R. J. Tomake, Assembly of the Vasculature and Its Regulation, Birkhäuser, Berlin, Germany, 2001.

[2] E. M. Wahl, L. V. Quintas, L. L. Lurie, and M. L. Gargano, "A graph theory analysis of renal glomerular microvascular networks," Microvascular Research, vol. 67, no. 3, pp. 223-230, 2004.

[3] D. A. Nordsletten, S. Blackett, M. D. Bentley, E. L. Ritman, and N. P. Smith, "Structural morphology of renal vasculature," American Journal of Physiology, vol. 291, no. 1, pp. H296-H309, 2006.

[4] M. Zamir, "Arterial branching within the confines of fractal Lsystem formalism," Journal of General Physiology, vol. 118, no. 3, pp. 267-275, 2001.

[5] A. Espinoza-Valdez, R. Femat, and F. C. Ordaz-Salazar, "A model for renal arterial branching based on graph theory," Mathematical Biosciences, vol. 225, no. 1, pp. 36-43, 2010.

[6] A. C. Guyton and J. E. Hall, Tratado de Fisiología Médica, McGraw-Hill, 10th edition, 2000, in Spanish.

[7] S. Patan, "Vasculogenesis and angiogenesis as mechanisms of vascular network formation, growth and remodeling," Journal of Neuro-Oncology, vol. 50, no. 1-2, pp. 1-15, 2000.

[8] M. Zamir and S. Phipps, "Morphometric analysis of the distributing vessels of the kidney," Canadian Journal of Physiology and Pharmacology, vol. 65, no. 12, pp. 2433-2440, 1987.

[9] Y. Gazit, D. A. Berk, M. Leunig et al., "Scale-invariant behavior and vascular network formation in normal and tumor tissue," Physical Review Letters, vol. 75, no. 12, pp. 2428-2431, 1995.

[10] Y. Gazit, J. W. Baish, N. Safabakhsh, M. Leunig, L. T. Baxter, and R. K. Jain, "Fractal characteristics of tumor vascular architecture during tumor growth and regression," Microcirculation, vol. 4, no. 4, pp. 395-402, 1997.

[11] J. Panico and P. Sterling, "Retinal neurons and vessels are not fractal but space-filling," Journal of Comparative Neurology, vol. 361, no. 3, pp. 479-490, 1995.

[12] P. G. Vico, S. Kyriacos, O. Heymans, S. Louryan, and L. Cartilier, "Dynamic study of the extraembryonic vascular network of the chick embryo by fractal analysis," Journal of Theoretical Biology, vol. 195, no. 4, pp. 525-532, 1998.

[13] C. Arlt, H. Schmid-Schönbein, and M. Baumann, "Measuring the fractal dimension of the microvascular network of the chorioallantoic membrane," Fractals, vol. 11, no. 2, pp. 205-212, 2003.

[14] S. Lorthois and F. Cassot, "Fractal analysis of vascular networks: insights from morphogenesis," Journal of Theoretical Biology, vol. 262, no. 4, pp. 416-433, 2010.

[15] E. Sabo, A. Boltenko, Y. Sova, A. Stein, S. Kleinhaus, and M. B. Resnick, "Microscopic analysis and significance of vascular architectural complexity in renal cell carcinoma," Clinical Cancer Research, vol. 7, no. 3, pp. 533-537, 2001.

[16] S. S. Cross, R. D. Start, P. B. Silcocks, A. D. Bull, D. W. K. Cotton, and J. C. E. Underwood, "Quantitation of the renal arterial tree by fractal analysis," Journal of Pathology, vol. 170, no. 4, pp. 479484, 1993.

[17] M. N. Nakatsu, R. C. A. Sainson, S. Pérez-del-Pulgar et al., "VEGF 121 and $\mathrm{VEGF}_{165}$ regulate blood vessel diameter through vascular endothelial growth factor receptor 2 in an in vitro angiogenesis model," Laboratory Investigation, vol. 83, no. 12, pp. 1873-1885, 2003.

[18] E. Gabryś, M. Rybaczuk, and A. Kędzia, "Fractal models of circulatory system. Symmetrical and asymmetrical approach comparison," Chaos, Solitons \& Fractals, vol. 24, no. 3, pp. 707715, 2005.

[19] M. Barnsley, Fractals Everywhere, Academic Press, 1988. 


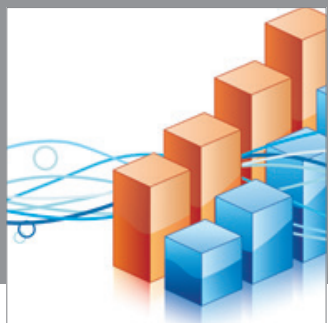

Advances in

Operations Research

mansans

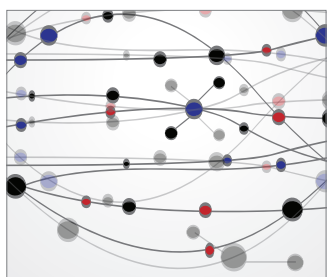

The Scientific World Journal
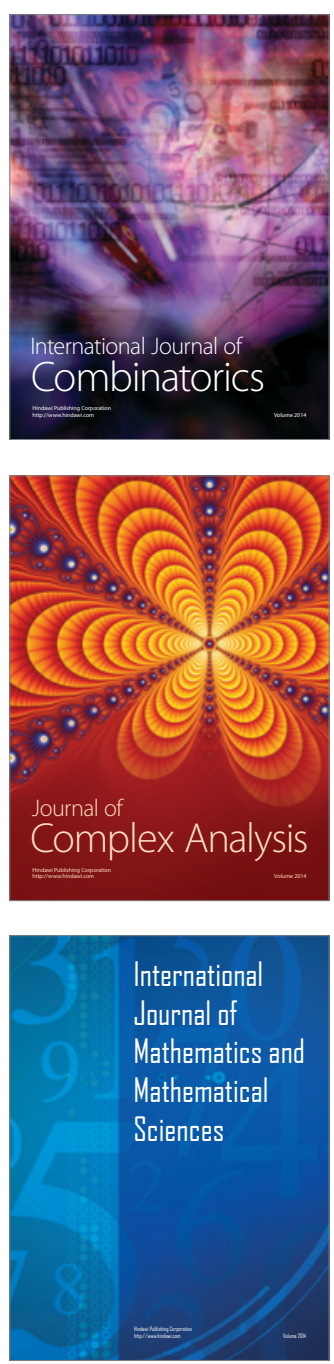
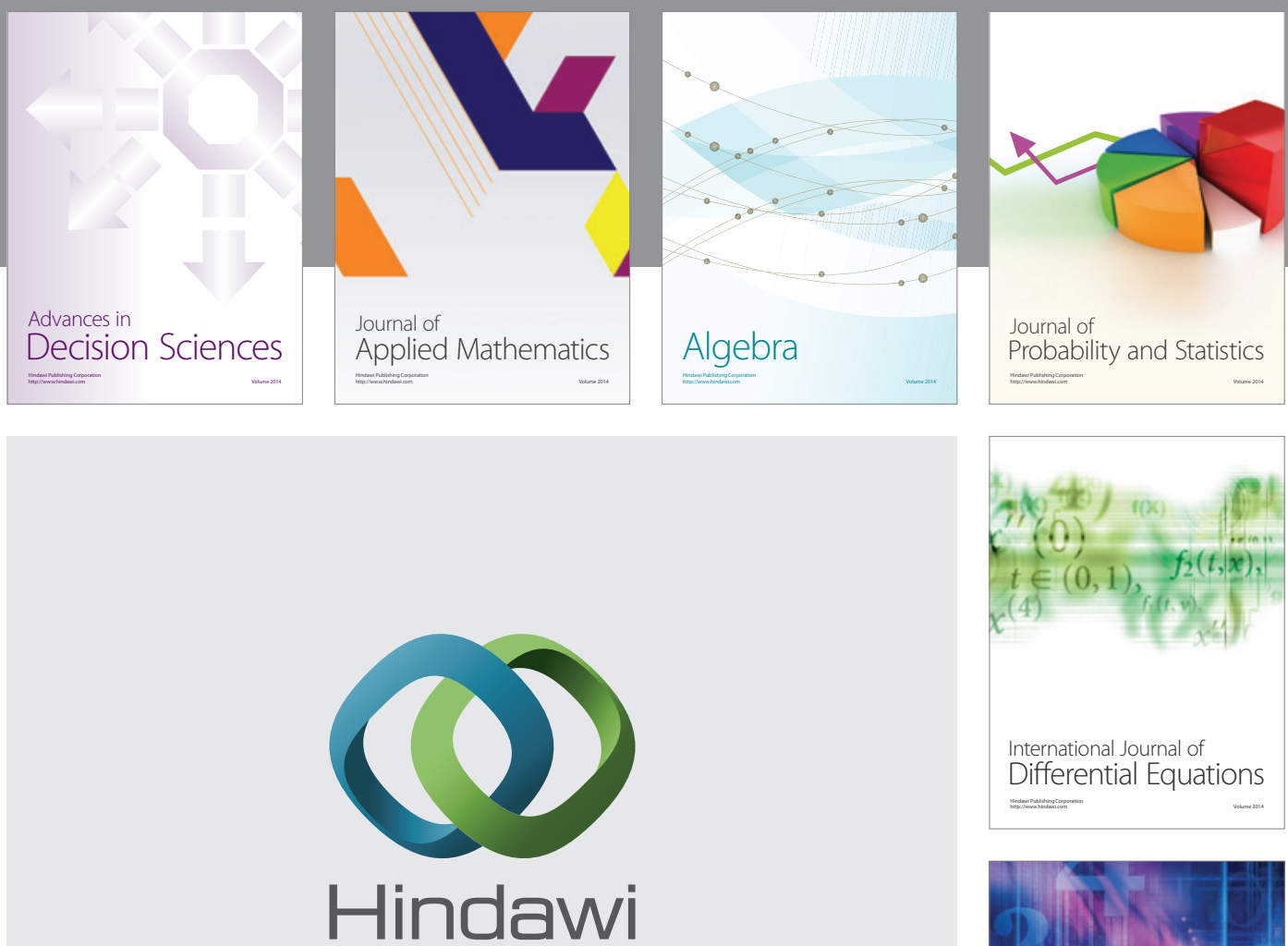

Submit your manuscripts at http://www.hindawi.com
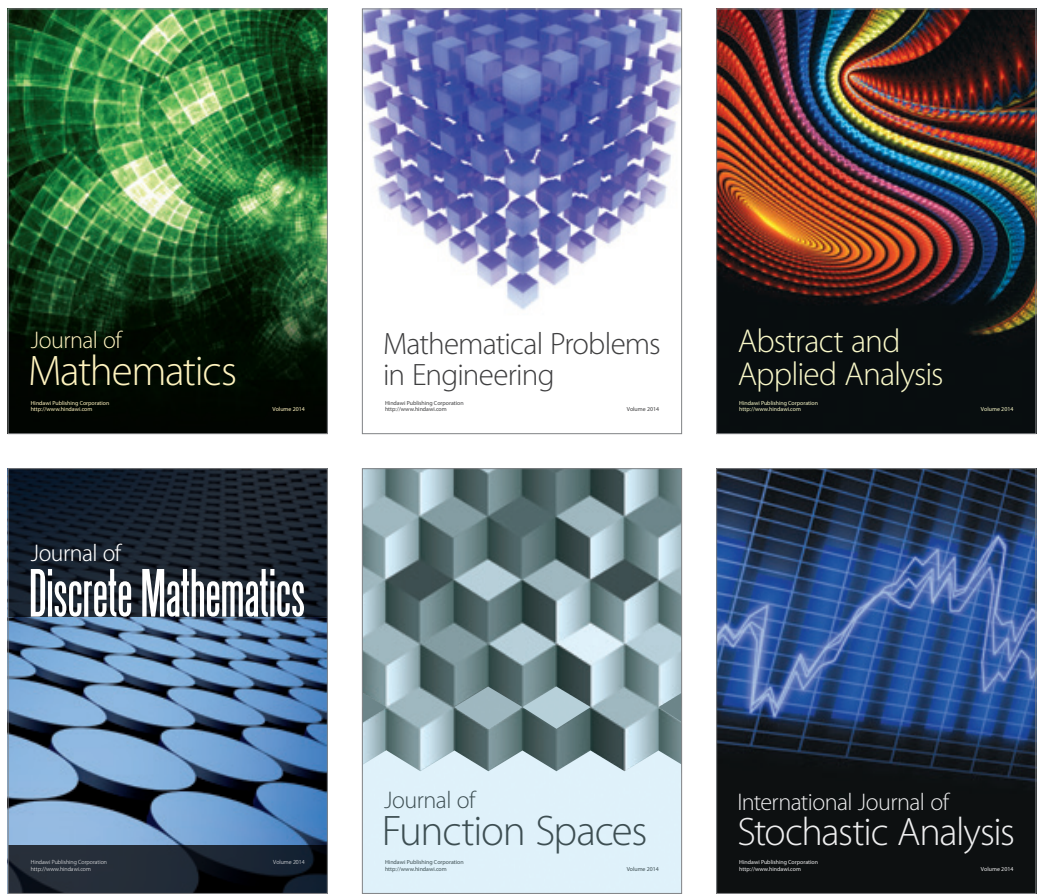

Journal of

Function Spaces

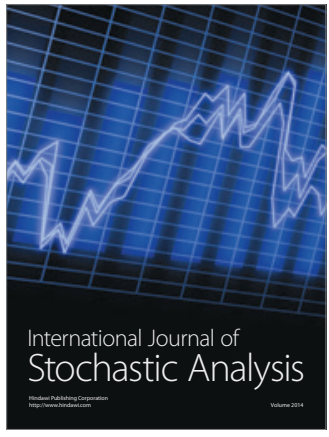

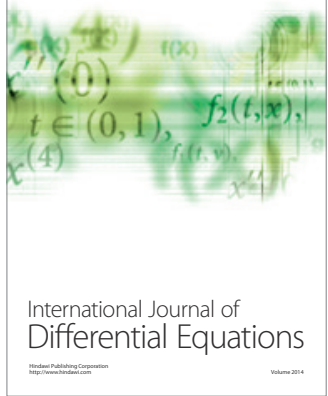
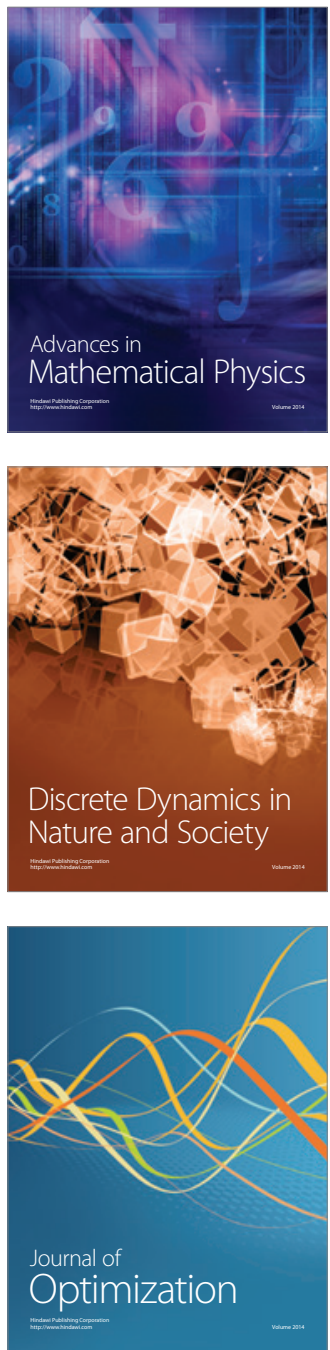